\title{
Hábitos informativos y participación online: un estudio sobre los y las jóvenes universitarios/as en Argentina
}

\section{Informative habits and online participation: a study about young}

undergraduates in Argentina

Hábitos de informação e participação online: um estudo sobre os e as jovens universitários/as na Argentina

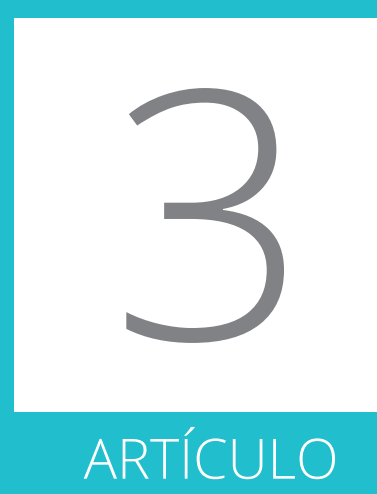

\section{(C) $(1) \Theta$}

\section{Raquel Tarullo}

Instituto de Política y Gobierno. Centro de Investigaciones Tecnológicas del Noroeste de Buenos Aires. CITnoba.UNNOBA. UNSAdA (Argentina)

Doctora en Ciencias Sociales y Humanas (UNQui). Profesora investigadora en la Universidad Nacional del Noroeste de la Provincia de Buenos Aires (UNNOBA) y en la Universidad Nacional de San Antonio de Areco (UNSAdA). Coordina el Proyecto I+D "Competencias digitales en la universidad y su impacto en las prácticas académicas y cívicas de estudiantes y profesores/ as" (Exp. 0556/2019-2021). En 2019 realizó una estancia de investigación en la Universidad de Valencia. Actualmente es Visiting Research Fellow en Goldsmiths, University of London.

mrtarullo@comunidad.unnoba.edu.ar orcid.org/0000-0003-2372-7571

RECIBIDO: 15 de enero de 2020 / ACEPTADO: 24 de abril de 2020

\section{Resumen}

Esta investigación explora los hábitos informativos y las prácticas de participación digital de los y las jóvenes universitarios/as en Argentina. Se utilizó una metodología mixta que combinó datos cuantitativos y cualitativos obtenidos a partir de encuestas ( $n=1243$ ) y entrevistas ( $n=132)$. Los resultados indican que los y las estudiantes se informan en dos etapas: se encuentran con la noticia incidentalmente, y luego profundizan en aquellas noticias con las cuales empatizan.
Prefieren entornos digitales, pero la televisión forma parte de su dieta informativa. Leen lo que otros comparten y comentan, pero prefieren mantenerse al margen de las conversaciones digitales.

\section{PALABRAS CLAVE}

Consumo de noticias, Estudiantes universitarios, Redes sociales, Participación política, Espacio público digital. 


\section{Abstract}

This research explores the information habits and digital participation practices of young undergraduates in Argentina. A mixed methodology was used that combined quantitative and qualitative data obtained from surveys $(n=1243)$ and interviews ( $n=132$ ). The results indicate that students are informed in two stages: they find the news incidentally and then delve into those news with which they empathize. They prefer digital environments, but television is part of their informational habits. They read what others share and comment, but prefer to stay away from digital conversations.

\section{KEYWORDS}

News consumption, Undergraduates, Social media, Political participation, Digital public space.

\section{Resumo}

Esta pesquisa explora os hábitos informacionais e as práticas de participação digital de jovens uni- versitários/as na Argentina. Foi utilizada uma metodologia mista que combinou dados quantitativos e qualitativos obtidos a partir de questionários ( $n=1243$ ) e entrevistas $(n=132)$. Os resultados indicam que os e as estudantes são informados em duas etapas: eles encontram as notícias por acaso e depois se aprofundam nestas notícias com as quais eles simpatizam. Eles preferem ambientes digitais, mas a televisão faz parte de sua dieta informacional. Eles leem o que os outros compartiIham e comentam, mas preferem ficar afastatos das conversas digitais.

\section{PALAVRAS-CHAVE}

Consumo de notícias, Estudantes universitários, Redes sociais, Participação política, Espaço público digital.

\section{INTRODUCCIÓN}

La incorporación de las redes sociales a las prácticas cotidianas ha ocasionado la modificación de gran parte de los procesos sociales, políticos, culturales y educativos, en un escenario versátil de mutación constante en el cual la interacción entre tecnología, usos y público se ve transformada de forma continua y vertiginosa. Uno de estos cambios está en relación con el consumo de noticias y la participación política. Por un lado, el paulatino abandono de los medios tradicionales como fuente de información acompañado de un creciente consumo de noticias en redes sociales es tema de análisis académico por las consecuencias que este cambio parece traer para la conformación de públicos informados, necesarios para la construcción de una sociedad anclada en valores democráticos
(Dahlgren, 2018; Pariser, 2017). Asimismo, las redes sociales han habilitado nuevos espacios y performances de participación, movilización y activismo político, antes reservado a la esfera urbana (Jenkins, et al., 2018) sobre todo en las comunidades más jóvenes (Tarullo \& García, 2020).

El porcentaje más alto de conectados en Argentina se concentra en la población más joven: casi el $80 \%$ de los y las argentinos/as de entre 15 y 34 años es un usuario diario de Internet y también en este rango etario se ubica el porcentaje más alto de usuarios/as de redes sociales (INDEC, 2017, 2018; Sistema de Información Cultural de la Argentina, 2017). Asimismo, Argentina se ubica tercero en el ranking de los países que más cantidad de horas son destinadas por su población a navegar en muros y perfiles (Kemp, 2018). 
Facebook, YouTube y WhatsApp son las redes sociales más utilizadas en Argentina (Hootsuite, 2018; Sistema de Información Cultural de la Argentina, 2017). Le siguen Instagram (27,30\%), Twitter (13,20\%), Snapchat (6,30\%) y Linkedln (2,10\%) (Hootsuite, 2018; Sistema de Información Cultural de la Argentina, 2017). Sin embargo, Instagram ha crecido sostenidamente en el último año entre adolescentes y jóvenes a partir de una arquitectura basada en la compartición de fotos y videos e historias que sólo sobreviven en el espacio digital por 24 horas (Alhabash \& Ma, 2017; Anderson \& Jiang, 2018; Newman et al., 2019).

Estos novedosos hábitos con y en los dispositivos tecnológicos modifican comportamientos y prácticas, entre ellas las relacionadas con el consumo de noticias y la participación política. La gran mayoría de los argentinos dice informarse en espacios online: el $86 \%$ afirma que es la web la fuente principal de noticias (Newman et al., 2019), porcentaje que incluye la búsqueda de contenidos en sitios informativos que solamente operan en formato online y también el consumo incidental de las noticias que circulan en las redes sociales (Antunovic et al., 2018; Serrano-Puche et al., 2018). En este sentido, un 63\% utiliza Facebook para informarse, mientras que WhatsApp es elegido por el 39\% de la población, Twitter por el 15\%, YouTube por el 25\%, Instagram por el $18 \%$ y Facebook Messenger por el 11\% (Newman et al., 2019). Instagram es la única red social que no sólo no ha disminuido el número de argentinos/as que se informan por esta vía, sino que además ha crecido cinco puntos respecto de 2018 (Newman et al., 2018; Newman et al., 2019).

En el último informe sobre consumos culturales digitales en el país, se afirma que el 35,40\% de los y las argentinas comenta y comparte contenidos, de cualquier tipo, en las redes sociales y más del 32, 80\% lee las publicaciones, pero no las comenta (Sistema de Información Cultural de la Argentina, 2017). Respecto de la interacción con el contenido noticioso, el 58\% de los y las usuarias de redes sociales comparte noticias por estas plataformas o por mail, mientras que el 31\% elige realizar comentarios sobre los contenidos de interés público (Newman et al., 2019)

En este escenario, esta investigación indaga en los hábitos informativos de estudiantes universitarios/as de Argentina para conocer sus fuentes de consumo noticioso y se pregunta si, cuando este grupo dice consumir noticias en espacios digitales, utiliza o no las herramientas de interacción que las redes sociales admiten, tales como la compartición de contenido y la posibilidad de expresar comentarios, prácticas que pueden ser entendidas como colaboradoras en la ampliación del espacio público y en la participación de la cosa pública (Dahlgren, 2018; Jenkins et al., 2018; Pereira et al., 2012).

\section{ANTECEDENTES EN LA TEMÁTICA}

Un importante cúmulo de investigaciones da cuenta del rol esencial que tiene el consumo de noticias para la vida democrática de una sociedad a partir de su incidencia en la participación social, cultural y política de los y las ciudadanos/ as en general (Bailey et al., 2014; Carpentier et al., 2013; Dahlgren, 2005) y de los más jóvenes en particular (Almansa-Martínez et al., 2013; Barredo Ibáñez et al., 2018; García Jiménez et al., 2018; Yuste, 2015).

Entre los diversos estudios que han servido como antecedente a esta investigación, se ubica la investigación de Antunovic et al. (2018) que concluye que la vigilancia, el acceso incidental a la noticia y el consumo directo de la misma a partir de distintos intereses y motiva- 
ciones, son los tres momentos que conforman las nuevas conductas informativas de los jóvenes que transcurren fundamentalmente en el espacio digital (Antunovic et al., 2018). El acceso a las noticias en espacios digitales, y a través de móviles, pero en convivencia con los medios tradicionales; forma parte de los hábitos informativos de los jóvenes españoles, en un esquema en el cual la rutina social y familiar incide en esta práctica cívica (Martínez Costa et al., 2019). Un estudio reciente realizado en Colombia sobre hábitos informativos y participación política entre estudiantes universitarios revela la escasa participación en espacios online y offline de este grupo social (Barredo Ibáñez et al., 2018). El acceso casual a la noticia desde las redes sociales y el acceso rápido a contenidos informativos en espacios digitales para chequear noticias de interés son parte de las conclusiones de la investigación de García Giménez et al. (2018). Un estudio sobre el consumo incidental de noticias en Facebook revela que el conocimiento previo que el o la usuario/a tiene sobre el tema de la información publicada es el principal motivo de involucramiento con las noticias que los y las usuarios/as encuentran de forma casual en ese medio social (Karnowski et al., 2017). En Argentina, el trabajo de Mitchelstein y Boczkowski (2017) examina el consumo incidental de las noticias a partir de los contenidos informativos que los y las jóvenes encuentran en sus muros cuando navegan en las redes digitales.

Consumir noticias sobre asuntos públicos está asociado con la conformación de un sujeto cívico comprometido con la participación en tópicos de interés general y también con el ejercicio de los deberes y derechos como ciudadanos y ciudadanas (Martinez \& Maldonado, 2017; Valenzuela, 2013). Asimismo, estar informado es uno de los pilares fundamentales para hablar y discutir sobre asuntos públicos en espacios de socialización (Brites, 2017; Campbell \& Kwak,
2011; Graber \& Dunaway, 2017; Habermas, 1990, 2006).

Con prácticas de consumo informativo en espacios digitales, la arquitectura de las redes sociales aporta herramientas de interacción y conversación que, a priori, admiten una ampliación de ese espacio de discusión habermasiano (Carpentier et al., 2013; Dahlberg, 2001; Dahlgren, 2018). Sobre esta cuestión, distintos estudios han indagado con resultados disímiles y hasta contradictorios: por un lado se ubican las investigaciones que sostienen que las redes sociales han colaborado en la ampliación de la participación cívica (Dahlgren, 2018; Papacharissi, 2015), mientras que otros estudios manifiestan que aquello que ocurre en los ámbitos digitales no tiene su corolario en el mundo offline, y que las prácticas en la dimensión online son propias del slacktivism o activismo de salón antes que manifestaciones reales de participación (Fuchs, 2017; Gladwell, 2010; Morozov, 2011), esquema que se complementa con el uso expandido del teléfono inteligente como dispositivo de acceso al mundo digital de las noticias (Deloitte, 2018). En este sentido, si bien para algunos el consumo de noticias a través del móvil inteligente es la única forma de acceso a contenidos informativos (Mossberger et al., 2013; Napoli \& Obar, 2014), para una gran mayoría la pantalla del celular limita la atención y la consulta de noticias se relaciona más con un "picoteo" de contenido antes que con la concentración y la atención que demandan este tipo publicaciones (Dunaway et al., 2018).

De la misma forma, investigaciones recientes indican que el creciente uso de Instagram y Snapchat por parte de los grupos etarios más jóvenes en detrimento de Facebook trae consecuencias esenciales respecto del consumo de noticias: si bien esta red social ha sido la autopista para el correr de noticias falsas, especialmente en períodos de campañas de comu- 
nicación electoral (Bounegru et al., 2017; Guess et al., 2018; Tucker et al., 2018), las interacciones que permite Facebook no las permite Instagram ni Snapchat, redes que en la actualidad reciben jóvenes y adolescentes migrados de Facebook (Tarullo, 2020) y cuya arquitectura prioriza la imagen por sobre el texto, y el emoji por sobre la palabra (Brake, 2018; Sampietro, 2019).

El objetivo de este estudio es indagar en los hábitos informativos de los y las estudiantes universitarios/as de Argentina a partir de las siguientes preguntas de investigación:

RQ1: ¿Cuáles son las fuentes de información del grupo poblacional estudiado?

RQ2: ¿Cuáles son los soportes que eligen para acceder a las noticias en el espacio digital?

RQ3: ¿Cuál es la frecuencia y la profundidad con la que se informan los y las estudiantes universitarios/as?

RQ4: ¿En qué medida el grupo estudiado interacciona con el contenido noticioso digital utilizando las herramientas que las redes sociales admiten?

\section{METODOLOGÍA}

Esta investigación se organiza a partir de una metodología mixta. Para la recolección de datos cuantitativos se llevó a cabo un cuestionario en formato digital y en una etapa posterior del estudio se realizaron entrevistas semiestructuradas con el fin de obtener información cualitativa que permita examinar y analizar el fenómeno de estudio en toda su complejidad. Los y las participantes fueron estudiantes universitarios/as de grado del centro de Argentina, tal como se detalla a continuación, que concurren a la Universidad Nacional del Noroeste de la provincia de Buenos Aires (UNNOBA), Ar- gentina. La investigación se radica en esta institución porque es una línea de estudio de un proyecto amplio que explora las competencias digitales de los y las estudiantes y profesores universitarios/as y la relación de esas competencias con sus prácticas académicas y cívicas. ${ }^{1}$ 'El alumnado de la UNNOBA está compuesto en más de un $85 \%$ por estudiantes que provienen de localidades del centro de Argentina (UNNOBA 2015, 2016, 2017).

Para la recolección de información cuantitativa, se constituyó una muestra ( $n=1243$ ) estratificada por escuela académica a partir de un muestreo aleatorio simple entre los y las estudiantes que concurrieron a clase en el período entre 20 de mayo y el 10 de junio de 2018.34 El 95\% de los participantes tienen entre 18 y 24 años, con una media resultante de 24 años y una moda de 21 años. La distribución por género de la muestra es de 39,70\% hombres y 59,50\% mujeres, mientras que un $0,80 \%$ prefirió no especificar.

Con un nivel de confianza del 95\%, los resultados obtenidos no son extrapolables a toda la población universitaria argentina, debido al carácter exploratorio de este estudio.

Para recoger los datos cualitativos, se diseñó una muestra no probabilística a partir de la técnica bola de nieve. La representatividad en el género y en la escuela académica de proce-

1. Esta investigación está enmarcada en el Proyecto I+D "Competencias digitales en la universidad y su impacto en las prácticas académicas y cívicas de estudiantes y profesores/as" (Exp. 0556/2019) financiado por la Universidad Nacional del Noroeste de la provincia de Buenos Aires, UNNOBA.

2. La UNNOBA está conformada por las siguientes escuelas académica: Tecnología, Agraria, Naturales y Ambientales, Económicas y Jurídicas y el Instituto de Desarrollo Humano. 
dencia de estudio de las y los encuestados/as, se logró a partir de atender, en una primera instancia, un conjunto por conveniencia de un alumno y una alumna por cada escuela. Esta muestra inicial de ocho estudiantes en total resultó el comienzo del muestreo lineal para acceder de este modo a la muestra definitiva de n=132 entrevistas. La mitad de las entrevistadas se identificó como mujer y la otra mitad como varón.

\subsection{INSTRUMENTO Y VARIABLES}

\subsubsection{EL CUESTIONARIO}

Con el fin de evaluar la pertinencia de las preguntas incluidas en el cuestionario y la conveniencia del formato digital de la encuesta como herramienta de recolección de datos, se testeó el diseño de la herramienta en un grupo de 30 estudiantes $(\mathrm{N}=30)$, seleccionados a partir de un muestreo por conveniencia. Con los resultados obtenidos, se examinó detalladamente el cuestionario para realizar los ajustes necesarios, se corrigieron preguntas y se precisaron las opciones de respuesta. Finalmente, la encuesta diseñada incluyó preguntas cerradas de respuesta única y sobre aspectos objetivos relacionados con los hábitos informativos de los universitarios, la identificación de las redes sociales como fuente de noticias y la utilización parte de las y los participantes de las herramientas de interacción, tales como compartir y comentar, siempre en relación con los contenidos noticiosos que el grupo participante consume en los espacios digitales. Los ejes temáticos incluidos en el cuestionario que respondieron los participantes estuvieron en relación con:

- Fuentes para el acceso a las noticias.
- El dispositivo/soporte utilizado para acceder a las noticias digitales

- Frecuencia en el consumo de noticias

- Profundidad en el consumo de noticias

- Consumo de noticias compartidas por los contactos

- Compartición de noticias en redes sociales

En todas las preguntas incluidas, el abanico de opciones fue construido a partir de las respuestas obtenidas en la prueba realizada en la instancia anterior a la aplicación definitiva del cuestionario a la muestra de estudio ( $\mathrm{N}=1243)$

Luego de que el cuestionario fuera expuesto por el equipo de investigación en momentos previos al dictado de clase, los y las jóvenes contestaron el cuestionario, en formato digital y de forma anónima, a través de sus teléfonos móviles; durante el período comprendido entre el 20 de mayo y el 10 de junio de 2018. El tiempo de respuesta fue entre 7 y 10 minutos.

\subsubsection{LA ENTREVISTA}

Para las entrevistas, se utilizó una guía orientadora para los y las entrevistadores con preguntas exploratorias sobre un abanico de temáticas propias del proyecto de investigación y de las cuales surgen los resultados incluidos en este artículo.

Las entrevistas, ejecutadas por esta autora y otros diez integrantes del grupo de estudio, fueron realizadas cara a cara, y grabadas previo asentimiento oral de los y las participantes. El promedio de duración de las entrevistas fue de 34 minutos. En una segunda etapa fueron desgrabadas en su totalidad por los y las participantes del proyecto. Para garantizar el anonimato, se utilizaron nombres de fantasía. Las entrevistas fueron realizadas en los espacios 
institucionales comunes, tales como pasillos, biblioteca, comedor y otros lugares de encuentro de la comunidad académica. La estrategia del muestreo fue por saturación de la información recolectada antes que en la representación del número de individuos determinados (Mejía Navarrete, 2000)

Con la lectura de las transcripciones se empezó con el análisis de las entrevistas. A partir de esta codificación individual se identificaron temáticas recurrentes respecto de los hábitos informativos que los y las jóvenes protagonizan en los espacios digitales como así también la participación desplegada por este grupo en el espacio digital.

En ambos instrumentos se indicó que se entendía por noticia a "toda información acerca de eventos y temas que implican algo más que solo la vida de sus familiares o amigos" (MESO, 2018, p.16).

\section{RESULTADOS}

Respecto de la RQ1 sobre cuál es la fuente que los y las estudiantes universitarios/as eligen para informarse, los datos obtenidos en este estudio indican que el 46,10\% ( $n=573$ ) prefiere los entornos digitales para acceder a contenidos informativos y esta performance incluye tanto el encuentro incidental con las noticias mientras consulta y navega en los muros de las redes sociales (Karnowski et al., 2017; MitcheIstein \& Boczkowski, 2017); como en el hecho de indagar en los detalles de aquellos contenidos informativos que le son de interés y cuyo primer acceso pudo darse a partir de distintos canales: publicidades digitales, notificaciones, un posteo compartido por sus contactos o una noticia televisiva, práctica observada en estudios anteriores (Antunovic et al., 2018). Alicia, una estudiante de ingeniería explicó esta prác- tica, al comentar en la entrevista realizada en el marco de este estudio, que se informa "viendo twitter o en la televisión, y si hay alguna noticia que me interesa la busco por internet en algún noticiero online que lo explique mejor porque generalmente en la televisión hay veces que lo explican muy por arriba. Entonces, si me interesa, lo busco". Este primer acceso a la noticia, que en algunos estudios previos es considerado como un monitoreo rápido y vigilante (García Jiménez et al., 2018), mientras que otros lo describen como un consumo incidental de las noticias (Mitchelstein \& Boczkowski, 2017), lo sintetiza Melina, una estudiante de diseño cuando confiesa: "Me informo con lo que salte, con las publicidades en general. No sigo a ningún medio de comunicación, tipo Clarín, Infobae. Pero si me interesa eso que me llegó, entro y lo leo". Lo mismo le ocurre a Jazmín, estudiante de Abogacía: "generalmente no visito las páginas de noticias, sino que las noticias me aparecen en Facebook, en Instagram, o en las publicidades de Google en el teléfono. Si me interesa, me voy metiendo, pero yo no las busco particularmente en un sitio online, las encuentro".

Los medios tradicionales siguen siendo una opción para los y las estudiantes universitarios/as a la hora de mantenerse informados, aunque con porcentajes dispares: mientras que casi el $37,80 \%$ ( $n=470$ ) de los y las encuestados/as sigue prefiriendo la televisión para consumir noticias, la lectura de noticias en el diario en formato papel es una práctica informativa en la que solo se ve representado en el 1,80\% ( $n=22)$ de los y las universitarios/as. Estos resultados se acercan a los parámetros que se observan a nivel mundial: de los medios tradicionales, mientras que el diario y la radio han perdido audiencia, en parte debido a la migración de sus seguidores a las versiones digitales de estos medios, es la televisión la que aún mantiene 
la atención del público en lo que a rutinas informativas respecta (Newman et al., 2019).

Mirar el noticiero televisivo del mediodía y de la noche se mantiene como hábito informativo familiar entre el grupo poblacional estudiado, pesar de haberse modificado el ecosistema de medios: "miro la tele en el almuerzo o en la cena, o sea de lunes a viernes siempre, y bueno, los fines de semana no, porque no hay noticiero", detalla Morena estudiante de ciencias económicas. El noticiero se mira en familia, una costumbre que se sostiene en el tiempo y que se ha observado en estudios previos (Martínez Costa et al., 2019). Jana, de informática lo dice: "A las 12 del mediodía miramos Telefe y a la noche, comiendo en familia, miramos la tele, el noticiero". Asimismo, la "tele" siempre encendida también incide en que sea alto el porcentaje de estudiantes que se informen por este medio: "Me informo con el noticiero a la hora de la cena, en familia, pero también porque es lo que está siempre puesto en casa", afirma Matías, estudiante de enfermería.

La respuesta de Nicolás, estudiante de ingeniería mecánica sintetiza esta convergencia de medios y prácticas para mantenerse informado: "la tele la pongo de fondo, principalmente en los canales de noticias, en TN, Telefé y en especial cuando ceno o almuerzo. Yo pongo y escucho y después en base a eso me voy a buscar algo que me interese en internet. Mi fuente de información es internet, porque está todo y podés leer muchas cosas"

Un dato significativo es el 5,80\% ( $n=72$ ) de los y las encuestados que se informa por WhatsApp, en un comportamiento de las audiencias en el consumo de noticias que ha comenzado a ser estudiado en la academia, en especial en escenarios electorales a partir de la incidencia de esta práctica en la difusión de noticias falsas y escenarios que colaboran en la desinformación del ciudadano (Bounegru et al., 2017; Guess et al., 2018; Tucker et al., 2018). Sin embargo, al indagar en las entrevistas sobre estos hábitos, los y las participantes refieren que cuando reciben noticias por WhatsApp, suelen desestimarlas o chequearlas: "Si me interesa investigo, profundizó y si no pasó de largo. A veces que son un chusmerío, no me interesa", afirma Damián, de abogacía. Alejo, de ingeniería, confiesa que suele recibir noticias por WhatsApp, pero son locales, de su pueblo, y relacionadas con accidentes de tránsito. María, de enfermería, cuando recibe noticias por WhatsApp están en relación con cuestiones relacionadas con la carrera: "algún descubrimiento, o vacuna, siempre relacionadas con el tema salud".

Quienes dicen no consumir noticias son el 8,90\% ( $n=110)$ de la muestra, dando cuenta de la importancia que este grupo poblacional le otorga al estar informado.

Respecto de la RQ2 que examina los soportes elegidos para acceder a las noticias en el espacio digital, los resultados indican que el teléfono inteligente es el dispositivo más usado por los y las estudiantes para acceder al mundo digital en general y al mundo de las noticias en particular. El 88,41\% ( $n=1090)$ prefiere este dispositivo para navegar online, mientras que el porcentaje restante se divide entre notebooks $(6,22 \%, n=77)$, computadoras de escritorio $(4,90 \%, n=61)$ y tabletas $(0,47 \%, n=5)$. Este uso casi universal del teléfono móvil, identificado en informes anteriores (INDEC, 2018), lo sintetiza Georgina, una alumna abogacía cuando afirma: "El teléfono es una extensión de mi cuerpo", "Es mi todo", confiesa, Pedro que estudia administración.

Es en este dispositivo en el cual el grupo poblacional estudiado accede a sus perfiles de redes sociales y desde donde se informa primeramente, y busca, ya en una segunda instancia 
más información sobre aquellos contenidos que motivan e interesan. Por eso, Juana, de enfermería, ubica el móvil como una compañía, que está con ella en todos lados y la deja navegar por todos lados, también. Así el móvil es esa parte del cuerpo que los más jóvenes usan para sus prácticas cotidianas entre las que se incluye el consumo informativo (Tarullo, 2020).

La pregunta de investigación sobre la frecuencia y la profundidad en el consumo de las noticias, los hallazgos de este estudio revelan que el $50,70 \%$ ( $n=630$ ) afirma consumir noticias todos los días, el 41,70\% ( $n=518$ ) algunos días a la semana y el 3,70\% ( $n=46)$ se informa sólo una vez por semana.

Asimismo, casi el 57,90\% ( $n=720$ ) lee la nota completa, el 25,30\% ( $n=314$ ) las cinco primeras líneas y el 13,50\% ( $n=166)$ solo el titular. Sin embargo, al indagar sobre esta práctica en las entrevistas, el consumo completo de la noticia está en relación con el interés particular, congruente con esa búsqueda primera del contenido noticioso que resulte afín a las motivaciones individuales. "Sí me interesa las leo hasta el final", dice Alberto, estudiante de abogacía. Lo mismo Lola: "Si la noticia me llega y me interesa, la busco. Y si la encuentro y me interesa, la leo entera. Si no, la dejo apenas me aburro".

Respecto de la RQ4, el 15,51\% ( $\mathrm{n}=192)$ comparte noticias con sus contactos en las redes sociales, mientras que el porcentaje asciende a más del doble $(33.36 \% \mathrm{n}=416)$ cuando se observa a quienes dicen no compartir contenido noticioso en estas plataformas. El 48,48\% $(n=602)$ del grupo poblacional estudiado dice que sólo lo hace a veces y en $2,65 \%(n=33)$ reveló que no lee noticias en redes sociales. Mientras que, respecto al consumo del contenido informativo compartido por los contactos en las redes sociales, el 14,30\% ( $n=178)$ prefiere no acceder a este contenido, mientras que el $82,90 \%$ ( $n=1030$ ) opta por consumir las noticias compartidas. Y si la compartición no es una práctica extendida entre los y las jóvenes, tampoco lo es la expresión de comentarios: Facundo, aunque comparte en Instagram a partir de capturas de pantallas que publica en las historias, dice que nunca comenta: "Comparto noticias, leo lo que otros opinan, pero no comparto. No me gusta". Andreína, de agronomía, prefiere los encuentros presenciales no virtuales para hacer comentarios y expresar su opinión: "en las reuniones con familiares y amigos hablamos de política, charlo mucho y me trato de informar con familiares y conocidos. Trato de sacar conclusiones y encontrar algo positivo en las noticias, pero no opino en las redes". También Pedro prefiere la presencialidad y la intimidad de sus contactos más cercanos para opinar: "no me genera compartir mi opinión sobre ciertas noticias. Por ahí las comento con mis amigos, tengo algunos amigos con los que puedo compartir "y viste lo que paso con tal...", pero en alguna reunión, cara a cara, no en las redes".

Y así como leer lo compartido es más habitual que compartir, el grupo estudiado dice leer los comentarios, pero prefiere no opinar. Y la cuestión de leer opiniones de otros están en relación con dos temas: curiosidad y diversión. Ernestina, de la carrera de abogacía, dice que no le interesa opinar públicamente pero que sí lee los comentarios que otros dejan: "para ver qué opina la gente en cuanto a esa cuestión, las miradas que hay sobre un tema". Jana lee para divertirse: "Sí, y me rio muchísimo de los comentarios. Creo que pierdo más tiempo ahí que leyendo la noticia, los leo por diversión". Lo mismo hace Katia porque dice "que siempre hay un descolgado que te publica un chiste en el medio".

Paradójicamente, los motivos para no participar en discusiones y conversaciones del ám- 
bito público en los espacios digitales están en relación con la violencia que observan en la mayoría de las interacciones: "la gente se pelea mucho, son muy violentos. No quiero pasar por eso", manifestó Juana, estudiante de enfermería. "Una vez intenté, dejé un comentario en Twitter, pero lo borré. Me empezaron a bardear [agredir] tanto que me fui", confesó José.

\section{DISCUSIÓN Y CONCLUSIONES}

Los y las estudiantes universitarios/as del centro de Argentina acceden a las noticias en dos etapas (Antunovic et al., 2018). En la primera, se encuentran con las noticias de forma incidental en los muros de sus redes sociales (Mitchelstein \& Boczkowski, 2017; Serrano-Puche et al., 2018), también reciben notificaciones es sus dispositivos móviles, se les ofrece una publicidad de algún medio periodístico mientras navegan por los entornos digitales o las ven en el noticiero televisivo. Estas performances originan que los y las jóvenes aquí estudiados consideren que se informan diariamente, aunque esta no sea una práctica que ellas y ellos realicen en forma consciente ni reflexiva, al menos en este primer momento. En una segunda fase, si el contenido de la noticia encontrada en estas navegaciones o en el noticiero es afín a sus intereses, se activa una búsqueda consecuente con estas motivaciones (Antunovic et al., 2018), sí atenta y reflexiva.

En esta dieta informativa en dos pasos, la televisión sigue siendo protagonista, hecho constatado por otros estudios (Antunovic et al., 2018; Martínez Costa et al., 2019). Los y las jóvenes continúan reproduciendo un hábito que resiste al paso de los años y a las modificaciones de los ecosistemas mediáticos: el noticiero televisivo se mira en familia y mientras se almuerza y se cena. Sin embargo, esa práctica se complementa con la segunda etapa de la rutina informativa del grupo estudiado, caracterizada por la búsqueda de aquellos temas noticiosos con los cuales los y las estudiantes dicen sentirse atraídos o motivados. Queda para futuros estudios saber cuáles son esas temáticas y si el tema incide en el canal elegido para la búsqueda.

Tal como se observó en estudios anteriores sobre hábitos informativos y participación política de estudiantes universitarios (Barredo Ibáñez et al., 2018), la utilización de las herramientas de interacción digital no es frecuente en el grupo estudiado, al menos en el caso de los contenidos informativos. Esta relación dispar entre quienes consumen las noticias que comparten sus contactos en alguna de las redes sociales que utilizan habitualmente y la práctica de sí compartir parece indicar que los y las jóvenes universitarias continúan con hábitos informativos que refieren a medios tradicionales, a partir de un modelo de comunicación en el cual el público se mantiene en su rol de receptor, a pesar de encontrarse con dispositivos y plataformas que posibilitan el intercambio de contenidos y de roles (Graham et al., 2013; Graham et al., 2014).

Esta performance de observación pasiva antes que de acción fue también identificada en el abordaje cualitativo al indagar en la participación de los y las jóvenes a partir de comentar los contenidos informativos: los y las estudiantes leen lo que otros escriben, comentan y opinan en las redes sociales sobre cuestiones de interés público, pero eligen no hacer públicos sus propios comentarios. Y si bien usan las redes para sus prácticas cotidianas (INDEC, 2018), compartir visiones y emitir comentarios, queda para la esfera presencial, en reuniones y encuentros, con un círculo íntimo de amigos y familiares, donde no exista la agresión ni dis- 
cusiones violentas que resultan ser expulsoras e inhibidoras de prácticas de participación las y los jóvenes en entornos digitales. Nos preguntamos, a la luz de estos resultados, si el momento del noticiero televisivo en familia, resulta ser el encuentro en el cual se comparten comentarios y se hacen públicas las opiniones. Esta inquietud puede motivar futuros estudios que permitan seguir entendiendo el fenómeno aquí abordado.

Al ser el teléfono inteligente la pantalla a partir de la cual los y las jóvenes ingresan al mundo de las noticias, resultaría enriquecedor indagar de qué manera la atención con la que se accede al contenido es moldeada a partir del uso de estos dispositivos para reconocer el grado de incidencia del móvil en la atención aportada por el y la estudiante universitario/a al análisis e interpretación de las noticias relacionadas con los asuntos públicos de interés común (Dunaway et al., 2018).

Entonces, por un lado, el interés particular en la conformación de una dieta informativa personal e individual a partir de ir en busca de aquellos contenidos con los cuales se empatiza, más la escasa participación e interacción digital con contenidos informativos pone en discusión el rol de las redes sociales en la ampliación del espacio público (Couldry et al., 2014; Dahlgren, 2011). Resta indagar en futuras investigaciones cuáles son esos temas con los cuáles los y las jóvenes empatizan, y cuántos de estos coinciden, o no, con temáticas de interés público, atendiendo a la necesidad de estar informado para colaborar en la construcción de una sociedad anclada en valores democráticos (Bauman, 2012; Couldry et al., 2014; Pariser, 2017).

Este estudio tiene sus limitaciones. Por el carácter exploratorio del mismo, sus resultados no son extrapolables a toda la población universitaria argentina. Por otra parte, sería enriquecedor estudiar en profundidad otras formas de participación política en entornos digitales de los y las jóvenes, más allá de las aquí estudiadas. Y también ahondar en las cuestiones relacionadas con las conversaciones sobre cuestiones de interés público que los y las jóvenes dijeron protagonizar en reuniones y encuentros presenciales y su posible interacción con las participaciones digitales del grupo estudiado. 


\section{REFERENCIAS}

Alhabash, S., \& Ma, M. (2017). A Tale of Four Platforms: Motivations and Uses of Facebook, Twitter, Instagram, and Snapchat Among College Students? Social Media and Society, 3(1). https://doi. org/10.1177/2056305117691544

Almansa-Martínez, A., Fonseca, O., \& Castillo-Esparcia, A. (2013). Social networks and young people. Comparative study of facebook between CoIombia and Spain. Comunicar, 20(40), 127-134. https://doi.org/10.3916/ C40-2013-03-03

Anderson, M., \& Jiang, J. (2018). Teens, Social Media \& Technology 2018. Pew Research Center.

Antunovic, D., Parsons, P., \& Cooke, T. R. (2018). 'Checking' and googling: Stages of news consumption among young adults. Journalism, 19(5), 632-648. https://doi.org/10.1177/1464884916663625

Bailey, M., Carpentier, N., Fenton, N., Jenkins, H., Lothian, A., \& Qiu, J. L. (2014). Participations: Dialogues on the Participatory Promise of Contemporary Culture and Politics. International Journal of Communication, 8, 1129-1151.

Barredo Ibáñez, D., de la Garza Montemayor, D. J., \& Días, D. L. (2018). The relationship between the consumption of digital media, participation and political efficacy. A study about young university students in CoIombia. Revista Latina de Comunicacion Social, 73, 945-960. https://doi. org/10.4185/RLCS-2018-1290

Bauman, Z. (2012). A Chronicle of crisis 2011-2016. Social Europe Editionts.

Bounegru, L., Gray, J., Tommaso, V., \& Mauri, M. (2017). A field guide to fake news and other information disorders. Public Data Lab. Amsterdam.

Brake, D. (2018). The social implications of teens leaving Facebook. The Conversation. https://theconversation.com/the-social-implications-of-teens-leaving-facebook-99362

Brites, M. J. (2017). Young people and daily life contexts of news appropriation Jovens e contextos quotidianos de consumo e apropriação de Jóvenes y contextos cotidianos de consumo y apropiación de noticias. Cuadernos.Info, (40), 137-151. https://doi.org/10.7764/cdi.40.1059

Campbell, S. W., \& Kwak, N. (2011). Mobile communication and civic life: Linking patterns of use to civic and political engagement. Journal of Communication, 60(3), 536-555. https://doi.org/10.1111/j.14602466.2010.01496.x 
Carpentier, N., Dahlgren, P., \& Pasquali, F. (2013). Waves of media democratization: A brief history of contemporary participatory practices in the media sphere. Convergence: The International Journal of Research into New Media Technologies, 19(3), 287-294. https://doi. org/10.1177/1354856513486529

Couldry, N., Stephansen, H., Fotopoulou, A., MacDonald, R., Clark, W., \& Dickens, L. (2014). Digital citizenship? Narrative exchange and the changing terms of civic culture. Citizenship Studies, 18(6-7), 615-629. https://doi. org/10.1080/13621025.2013.865903

Dahlberg, L. (2001). Computer-mediated communication and the public sphere: A critical analysis. Journal of Computer-Mediated Communication, 7(1). https://doi.org/10.1111/j.1083-6101.2001.tb00137.x/full

Dahlgren, P. (2005). The Internet, Public Spheres, and Political Communication: Dispersion and Deliberation. In Political Communication (Vol. 22, pp. 147-162). Routledge. https://doi.org/10.1080/10584600590933160

Dahlgren, P. (2011). Parameters of online participation: conceptualising civic contingencies. Communication Management Quaterly, (21).

Dahlgren, P. (2018). La participación en línea en la esfera pública. Inmediaciones de La Comunicación, 13(1), 25-47. https://doi.org/10.18861/ ic.2018.13.1.2824

Deloitte. (2018). Consumo móvil en Argentina. https://www2.deloitte.com/content/dam/Deloitte/ar/Documents/technology-media-telecommunications/Argentina-Mobile-Consumer-Trends_Diciembre-2017.pdf

Dunaway, J., Searles, K., Sui, M., \& Paul, N. (2018). News Attention in a Mobile Era. Journal of Computer-Mediated Communication, 23(2), 107-124. https://doi.org/10.1093/jcmc/zmy004

Fuchs, C. (2017). Social Media, a critical introduction (Second Edi). Sage Publications.

García Jiménez, A., Tur-Viñes, V., \& Pastor Ruiz, Y. (2018). Consumo mediático de adolescentes y jóvenes. Noticias, contenidos audiovisuales y medición de audiencias. Revista ICONO14 Revista Científica de Comunicación y Tecnologías Emergentes, 16(1), 22. https://doi.org/10.7195/ri14. v16i1.1101

Gladwell, M. (2010). Small change. Why the revolution will not be tweeted. The New Yorker, 42-49.

Graber, D., \& Dunaway, J. (2017). Mass Media and American Politics. DC: CQ Press. 
Graham, T., Broersma, M., Hazelhoff, K., \& van 't Haar, G. (2013). Between broadcasting messages and interacting with voters: The use of Twitter during the 2010 UK general election campaign. Information, Communication \& Society, 16(5), 692-716. https://doi.org/10.1080/1369118X.2013.785581

Graham, T., Jackson, D., \& Broersma, M. (2014). New platform, old habits? Candidates' use of Twitter during the 2010 British and Dutch general election campaigns. New Media \& Society, online first. https://doi. org/10.1177/1461444814546728

Guess, A., Nyhan, B., \& Reifler, J. (2018). Selective exposure to misinformation: evidence from the consumption of fake news during the $2016 \mathrm{U}$. S. presidential campaign. European Journal of Communication, (682758), $1-34$.

Habermas, J. (1990). Historia y crítica de la opinión pública (4th ed.). Gustavo Gili.

Habermas, J. (2006). Political Communication in Media Society: Does Democracy Still Enjoy an Epistemic Dimension? The Impact of Normative Theory on Empirical Research. Communication Theory, 16(4), 411-426. https://doi.org/10.1111/j.1468-2885.2006.00280.x

Hootsuite. (2018). Digital in 2018 in Southern America. We Are Social. New York. https://es.slideshare.net/wearesocial/digital-in-2018-in-southernamerica-part-1-north-86863727

INDEC. (2017). Informes Técnicos Ciencia y tecnología. Acceso y uso de tecnologías de la información y la comunicación (Vol. 2).

INDEC. (2018). Accesos a Internet. Buenso Aires. www.indec.gob.ar/calendario. asp

Jenkins, H., Gamber-Thompson, L., Kligler-Vilenchik, N., Shresthova, S., \& Zimmerman, A. (2018). Any Media Necessary: Mapping Youth and Participatory Politics. NYUPress.

Karnowski, V., Kümpel, A. S., Leonhard, L., \& Leiner, D. J. (2017). From incidental news exposure to news engagement. How perceptions of the news post and news usage patterns influence engagement with news articles encountered on Facebook. Computers in Human Behavior, 76, 42-50. https://doi.org/10.1016/j.chb.2017.06.041

Kemp, S. (2018). Digital in 2018. Essential insights into Internet, social media, mobile and e-commerce use around the world. https://wearesocial. com/blog/2018/01/global-digital-report-2018

Martínez Costa, M., Serrano Puche, J., Portilla Manjón, I., \& Sánchez Blanco, C. (2019). La interacción de los jóvenes adultos con las noticias y la publicidad online. Comunicar : Revista Científica Iberoamericana de Comunicación y Educación, 19-28. https://doi.org/10.3916/c59-2019-02 
Martinez, J. de D., \& Maldonado, L. (2017). El efecto de la atención a noticias en la participación política de los jóvenes en el Área Metropolitana de Monterrey, México. Un estudio longitudinal. Cuadernos.Info, (40), 39-52. https://doi.org/10.7764/cdi.40.1095

Mejía Navarrete, J. (2000). El muestreo en la investigación cualitativa. Investigaciones Sociales, $N(5), 165-180$.

MESO. (2018). Especial Consumo de Información. Buenso Aires. http://meso. com.ar/wp-content/uploads/2018/11/12.-UdeSA-Especial-Medios.pdf

Mitchelstein, E., \& Boczkowski, P. J. (2017). Juventud, estatus y conexiones. Explicación del consumo incidental de noticias en redes sociales. Revista Mexicana de Opinión Pública, (24), 131. https://doi.org/10.22201/ fcpys.24484911e.2018.24.61647

Morozov, E. (2011). The Net Delusion. (Vol. 9). Penguin Books.

Mossberger, K., Tolbert, C. J., \& Franko, W. W. (2013). Digital cities: The Internet and the geography of opportunity. Oxford University Press.

Napoli, P., \& Obar, J. (2014). The emerging mobile Internet underclass: A critique of mobile Internet access. Information Society, 30(5), 323-334. https://doi.org/10.1080/01972243.2014.944726

Newman, N., Fletcher, R., Kalogeropoulos, A., Levy, D. A. L., \& Kleis Nielsen, R. (2018). Digital News Report 2018. https://reutersinstitute.politics.ox.ac. uk/sites/default/files/digital-news-report-2018.pdf

Newman, N., Richard Fletcher, W., Kalogeropoulos, A., \& Kleis Nielsen, R. (2019). Reuters Institute Digital News Report 2019. https://reutersinstitute.politics.ox.ac.uk/sites/default/files/2019-06/DNR_2019_FINAL_1. pdf

Papacharissi, Z. (2015). Affective Publics. Oxford University Press.

Pariser, E. (2017). El filtro burbuja: Cómo la web decide lo que leemos y lo que pensamos. Taurus.

Pereira, G. C., Furtado Rocha, M. C., \& Poplin, A. (2012). e-Participation : Social Media and the Public Space. Computational Science and Its Applications, 7333, 491-501. https://doi.org/10.1007/978-3-642-31125-3_37

Sampietro, A. (2019). Emoji and rapport management in Spanish WhatsApp chats. Journal of Pragmatics, 143, 109-120. https://doi.org/10.1016/J. PRAGMA.2019.02.009

Serrano-Puche, J., Fernández, C. B., \& Rodríguez Virgili, J. (2018). Información política y exposición incidental en las redes sociales : un análisis de Argentina, Chile, España y México. Doxa. Comunicación, 27, 19-42. https://doi.org/10.31921/doxacom.n27a1 
Sistema de Información Cultural de la Argentina. (2017). Encuesta Nacional de Consumos Culturales. Encuesta Nacional de Consumos Culturales 2017. https://www.sinca.gob.ar/Encuestas.aspx

Tarullo, R. (2020). ¿Por qué los y las jóvenes están en las redes sociales? Un análisis de sus motivaciones a partir de la teoría de usos y gratificaciones. Revista Prisma Social, (29). En prensa

Tarullo, R., \& García, M. (2020). Hashtivismo feminista en Instagram: \#NiñasNoMadres de @actrices.argentinas. Dígitos. Revista de Comunicación Digital, (6), 31-54. https://doi.org/10.7203/rd.v1i6.172

Tucker, J. A., Guess, A., Barbera, P., Vaccari, C., Siegel, A., Sanovich, S., Stukal, D., \& Nyhan, B. (2018). Social Media, Political Polarization, and Political Disinformation: A Review of the Scientific Literature. Ssrn, (March), 1-95. https://doi.org/10.2139/ssrn.3144139

UNNOBA (2015) UNNOBA. (2016). Informe Secretaría Académica de la Universidad Nacional del Noroeste de la Provincia de Buenos Aires. CEDI. Junín.

UNNOBA (2016) UNNOBA. (2016). Informe Secretaría Académica de la Universidad Nacional del Noroeste de la Provincia de Buenos Aires. CEDI. Junín.

UNNOBA (2017) UNNOBA. (2016). Informe Secretaría Académica de la Universidad Nacional del Noroeste de la Provincia de Buenos Aires. CEDI. Junín.

Valenzuela, S. (2013). Unpacking the Use of Social Media for Protest Behavior: The Roles of Information, Opinion Expression and Activism. American Behavioral Scientist, 57, 920-942. https://doi. org/10.1177/0002764213479375

Yuste, B. (2015). Las nuevas formas de consumir información de los jóvenes. Revista de Estudios de La Juventud, 108, 179-191. 